\title{
A PT-Invariant Potential With Complex QES Eigenvalues
}

\author{
Avinash Khare \\ Institute of Physics, Sachivalaya Marg, \\ Bhubaneswar-751005, India, \\ Email: khare@iopb.res.in \\ Bhabani Prasad Mandal \\ S.N. Bose National Center for Basic Sciences, \\ Block-JD; Sector-III; Salt Lake, \\ Calcutta-700 091, India. \\ Email:bpm@boson.bose.res.in
}

\begin{abstract}
We show that the quasi-exactly solvable eigenvalues of the Schrödinger equation for the PT-invariant potential $V(x)=-(\zeta \cosh 2 x-i M)^{2}$ are complex conjugate pairs in case the parameter $\mathrm{M}$ is an even integer while they are real in case $\mathrm{M}$ is an odd integer. We also show that whereas the PT symmetry is spontaneously broken in the former case, it is unbroken in the latter case.
\end{abstract}


In non-relativistic quantum mechanics, one usually chooses a real (Hermitian) potential so as to ensure the real energy eigenvalues of the corresponding Schrödinger equation. Few years ago, Bender and others [1,2] have studied several complex potentials which are invariant under the combined symmetry $P T$ and showed that even in all these cases, the energy eigenvalues of the Schrödinger equation are real so long as the $P T$ symmetry is not spontaneously broken. This seem to suggest that instead of Hermiticity, it may be enough to have PT-invariant Hamiltonian so as to have real energy eigenvalues. It must however be noted that so far, this is merely a conjecture which is being supported by several examples. Of course, even if this conjecture is true, there are several basic questions which will have to be addressed before one can take the PT-invariant potentials more seriously.

It must however be noted here that PT invariance is not sufficient to ensure the reality of spectrum. As has been noted by Bender and Boettcher [1], one has to ensure that the PT symmetry is not spontaneously broken. As an illustration, using extensive numerical and asymptotic studies, they have shown that the eigenvalues of the potential $-(i x)^{N},(N$ real) are real if $N \geq 2$ while there are finite number of real and infinite number of complex conjugate pairs of eigenvalues if $1<N<2$. They also showed that whereas PT symmetry is unbroken if $N \geq 2$, it is spontaneously broken if $1<N<2$.

Thus it is clearly of great importance to understand as to when the PT symmetry is spontaneously broken and when it remains unbroken. As a first step in that direction, it may be worthwhile to look for some analytically solvable PT-invariant potentials and try to understand the spontaneous breaking and nonbreaking of the PT symmetry. The purpose of this note is to study one such example. In particular, we consider the system described by the non-Hermitian but PT-invariant Hamiltonian $(\hbar=2 m=1)$

$$
H=p^{2}-(\zeta \cosh 2 x-i M)^{2},
$$

where the parameter $\zeta$ is real and parameter $M$ has only integer values. We show that the quasi-exactly solvable (QES) eigen values [3] of this Hamiltonian are complex conjugate pairs in case the parameter $\mathrm{M}$ is an even integer and that in this case the PT symmetry is 
indeed spontaneously broken. On the other hand, when $M$ is an odd integer then the QES eigenvalues of this Hamiltonian are real and precisely in this case the PT symmetry remains unbroken.

Let us first show that the above potential is PT-invariant. To that end, notice that under T reflection one replaces $i$ by $-i$ while under $\mathrm{P}$ reflection one replaces $x$ by $a-x$, where $a / 2$ is the origin about which one is performing the parity reflection. It is then easily checked that the Hamiltonian (1) is PT symmetric under the parity reflection $x \rightarrow i \pi / 2-x$.

We substitute

$$
\psi(x)=e^{i \frac{\zeta}{2} \cosh 2 x} \phi(x)
$$

in the Schroödinger equation $H \psi=E \psi$ with $H$ as given by Eq. (0.1) and obtain

$$
\phi^{\prime \prime}(x)+2 i \zeta \sinh 2 x \phi^{\prime}(x)+\left[\left(E-M^{2}+\zeta^{2}\right)-2 i(M-1) \zeta \cosh 2 x\right] \phi(x)=0 .
$$

Ordinarily, the boundary conditions that give the quantized energy levels are $\psi(x) \rightarrow 0$ as $|x| \rightarrow \infty$ on the real axis. However, in the present case, we have to continue the eigenvalue problem into the complex-x plane 4 . On putting, $x=u+i v$ where $u, v$ are real, it is easy to see that for $u>0$ the above boundary condition is satisfied so long as $-\pi<v<-\pi / 2(\bmod \pi)$ while for $u<0$ it is satisfied if $-\pi / 2<v<0(\bmod \pi)$.

On further substituting [5]

$$
z=\cosh 2 x-1 ; \quad \phi=z^{s} \sum_{n=0}^{\infty} \frac{R_{n}(E)}{n !}\left(\frac{z+2}{2}\right)^{\frac{n}{2}},
$$

we obtain the three-term recursion relation $(n \geq 0)$

$$
\begin{gathered}
R_{n+2}(E)-\left[n^{2}+4(s-i \zeta) n+4 s^{2}+E-M^{2}+\zeta^{2}+2 i(M-1) \zeta\right] R_{n}(E) \\
+\quad 4 i \zeta[M+1-2 s-n] n(n-1) R_{n-2}(E)=0
\end{gathered}
$$

provided $2 s^{2}=s$ i.e. either $s=0$ or $s=\frac{1}{2}$. Thus we have two sets of independent solutions; one for $s=0$ and other for $s=\frac{1}{2}$. Note that the parameter $s$ is not contained in the potential and this is perhaps related to the fact that for any (integer) $\mathrm{M}(>1)$, the QES solutions corresponding to both even and odd number of nodes are obtained. 
From Eq. (0.5) we observe that the even and odd polynomials $R_{n}(E)$ do not mix with each other and hence we have two separate three-term recursion relations depending on whether $n$ is odd or even. In particular, it is easily shown that the three-term recursion relations corresponding to the even and odd $n$ cases respectively are given by $(n \geq 1)$

$$
\begin{aligned}
P_{n}(E) & -\left[4 n^{2}+8 n(s-i \zeta-1)+4 s^{2}-8 s+4+6 i \zeta+E-(M-i \zeta)^{2}\right] P_{n-1}(E) \\
& +8 i \zeta(n-1)(2 n-3)(M+3-2 s-2 n) P_{n-2}=0 \\
Q_{n}(E)- & {\left[4 n^{2}+4 n(2 s-2 i \zeta-1)+4 s^{2}-4 s+1+2 i \zeta+E-(M-i \zeta)^{2}\right] Q_{n-1}(E) } \\
+ & 8 i \zeta(n-1)(2 n-1)(M+2-2 s-2 n) Q_{n-2}=0,
\end{aligned}
$$

with $P_{0}(E)=1, Q_{0}(E)=1$.

We have studied the properties of the weakly orthogonal polynomials $P_{n}(E)$ and $Q_{n}(E)$ and we find that many of these are similar to the Bender-Dunne polynomials [6]. Further, we find that for integer values of $M$, there are $M$ QES eigen values and they are complex conjugate pairs if $M$ is an even integer $(\mathrm{M}=2,4, \ldots)$ while they are real if $M$ is an odd integer (provided $|\zeta| \leq \zeta_{c}$, where $\zeta_{c}$ is a function of $\left.M\right)$. In particular, if $M=2 k+2(2 k+1)$ $(k=0,1, \ldots)$, then $k+1$ levels are obtained from the zeros of the orthogonal polynomial $P_{k+1}(E)$ while $k+1(k)$ levels are obtained from the zeros of $Q_{k+1}\left(Q_{k}\right)$ which we term as the critical polynomials. Further, all higher $P_{n}$ and $Q_{n}$ polynomials exhibit factorization property.

Let us first consider few even integer values of $M$ and show explicitly that the QES eigenvalues are complex conjugate pairs and further the PT symmetry is spontaneously broken. We start from $\mathrm{M}=2$ for which case the critical polynomials $P_{1}(E)($ with $s=1 / 2)$ and $Q_{1}(E)$ (with $s=0$ ) are given by

$$
P_{1}(E)=E-3+\zeta^{2}+2 i \zeta, \quad Q_{1}(E)=E-3+\zeta^{2}-2 i \zeta
$$

hence the two QES complex conjugate pair of energy eigenvalues are

$$
E_{ \pm}=3-\zeta^{2} \pm 2 i \zeta
$$


The corresponding eigenfunctions are as given by eq. (0.2) with

$$
\phi_{+}=\cosh x, \phi_{-}=\sinh x .
$$

Now under the PT transformation as defined above, $\cosh x \rightarrow-i \sinh x, \sinh x \rightarrow i \cosh x$ while $i \cosh 2 x$ remains invariant so that the two wave functions are not invariant under PT, i.e. in this case (where the eigenvalues are complex conjugate pair), the PT symmetry is spontaneously broken.

For $M=4$, the critical polynomials are

$$
\begin{aligned}
& P_{2}(E)=\epsilon^{2}+(10+4 i \zeta) \epsilon+3\left(4 \zeta^{2}+12 i \zeta+3\right), \\
& Q_{2}(E)=\epsilon^{2}+(10-4 i \zeta) \epsilon+3\left(4 \zeta^{2}-12 i \zeta+3\right),
\end{aligned}
$$

where $\epsilon=E-16+\zeta^{2}$. Hence the four (two complex conjugate pair of) eigenvalues are

$$
\begin{aligned}
& E_{ \pm}^{1}=11-\zeta^{2}-2 i \zeta \pm 4 \sqrt{1-i \zeta-\zeta^{2}} \\
& E_{ \pm}^{2}=11-\zeta^{2}+2 i \zeta \pm 4 \sqrt{1+i \zeta-\zeta^{2}}
\end{aligned}
$$

It is easily checked that the corresponding eigenfunctions are

$$
\begin{aligned}
& \phi_{ \pm}^{1}=A \sinh 3 x+B \sinh x, \frac{B}{A}=\frac{E-7+\zeta^{2}}{2 i \zeta}, \\
& \phi_{ \pm}^{1}=A \cosh 3 x+B \cosh x, \frac{B}{A}=\frac{E-7+\zeta^{2}}{2 i \zeta},
\end{aligned}
$$

so that in this case also the PT symmetry is spontaneously broken. It is easily checked that this is also true for all higher even integer values of $\mathrm{M}$.

On the other hand, if $M$ is an odd integer, then the eigenvalues are real provided $\zeta^{2} \leq \zeta_{c}^{2}$. For example, for $\mathrm{M}=1$, the critical polynomial is $P_{1}(E)=E-1+\zeta^{2}$ so that the QES level is at $E=1-\zeta^{2}$. In this case the eigenfunction is

$$
\phi=\text { constant }
$$


so that the corresponding $\psi$ is indeed an eigenfunction of PT with eigenvalue 1 and hence PT symmetry remains unbroken.

For $M=3$, the critical polynomials are

$$
P_{2}(E)=\epsilon^{2}+4 \epsilon+16 \zeta^{2}, \quad Q_{1}(E)=\epsilon+4,
$$

where $\epsilon=E-9+\zeta^{2}$. Thus the three QES eigenvalues are

$$
E=5-\zeta^{2}, \quad E_{ \pm}=7-\zeta^{2} \pm 2 \sqrt{1-4 \zeta^{2}}
$$

These energy levels are real if $\zeta^{2} \leq \zeta_{c}^{2}=1 / 4$ and at $\zeta=\zeta_{c}$, the two highest QES levels are degenerate. The corresponding eigenfunctions are

$$
\phi=\sinh 2 x, \phi_{ \pm}=A \cosh 2 x+i B
$$

where

$$
\frac{B}{A}=\frac{4 \zeta}{E-9+\zeta^{2}}
$$

It is easily checked that the corresponding wavefunction $\psi$ is indeed an eigenfunctions of PT with eigenvalue 1 while the other two wavefunctions $\left(\psi_{ \pm}\right)$are eigenfunctions with eigenvalue -1. Thus the PT symmetry remains unbroken. In fact, it is easily checked that this is indeed so for all higher odd integer values of $\mathrm{M}$ [7].

It is worth pointing out that if following Finkel et al. [8], we change the variable, $z=e^{2 x}$ and make the gauge transformation

$$
\mu(z)=z^{\frac{1-M}{2}} e^{\frac{i \zeta}{4}\left(z+\frac{1}{z}\right)}
$$

in the Hamiltonian (1) to map it to a differential operator $H_{g}$ (called as gauged Hamiltonian), then this gauged Hamiltonian can be expressed in terms of the generators of the $S l(2, R)$ by

$$
H_{g}(z)=-4 J_{0}^{2}+2 i \zeta\left(J_{-}-J_{+}\right)-c^{*},
$$

where $c^{*}=\zeta^{2}-M^{2}$ while the generators $J_{+,-, 0}$ are given by 


$$
J_{+}=\frac{\partial}{\partial z}, J_{0}=z \frac{\partial}{\partial z}-\frac{n}{2}, J_{-}=z^{2} \frac{\partial}{\partial z}-n z
$$

Further, in this case the norms and the weight functions of the corresponding orthogonal polynomials are real. Of course, as expected, the QES eigenvalues remain unchanged.

Finally, using the anti-isospectral transformation of Krajewska et al. [9], it follows that the QES eigenvalues and eigenfunctions of the PT-invariant potential (with parity reflection $\theta \rightarrow \pi / 2-\theta)$

$$
V(\theta)=(\zeta \cos 2 \theta-i M)^{2}
$$

are related to those of the potential in Eq. (1) by

$$
\bar{E}_{k}=-E_{M-1-k}, \bar{\psi}_{k}(\theta)=\psi_{M-1-k}(i x) .
$$

In particular, the QES eigenvalues of this periodic potential are real if $\mathrm{M}$ is an odd integer and further it is easily shown that the PT symmetry remains unbroken in this case. On the other hand, when $\mathrm{M}$ is an even integer, then the eigenvalues are complex conjugate pairs and PT symmetry is spontaneously broken. But in any case, the eigenstates are unacceptable in case $\mathrm{M}$ is an even integer [5] since the corresponding eigenfunctions do not satisfy the periodicity property of the above potential.

It would be worthwhile to explore as to when is PT symmetry likely to remain unbroken and when is it spontaneously broken.

Acknowledgement: We are grateful to the referee Prof. Carl M. Bender for pointing out the PT-invariance of the potentials discussed in this paper and reminding about the connection between the reality of the spectrum and the PT symmetry being unbroken. 


\section{REFERENCES}

[1] C.M. Bender and S. Boettcher, Phys. Rev. Lett. 80, 5243 (1998).

[2] C.M. Bender and S. Boettcher, J. Phys. A31, L273 (1998); C.M. Bender, S. Boettcher, and P.N. Meisinger, J. Math. Phys. 40, 2210 (1999); C.M. Bender, S. Boettcher, H.F. Jones and Van M. Savage, quant-ph/9906057; C.M. Bender and G. V. Dunne, J. Math. Phys. 40, 4616 (1999); C.M. Bender, G.V. Dunne and P.N. Meisinger, Phys. Lett A252, 272 (1999); C.M. Bender, K.A. Milton and P.N. Meisinger J. Math. Phys 402201 , (1999); C.M. Bender and K.A. Milton , hep-th/9802184; F.M. Fernandez, R. Guardiola, J. Ros and M. Znojil, J. Phys. A32, 3105 (1999); M. Znojil, J. Phys. A32, 4563 (1999); Phys. Lett. A264, 108 (1999); F. Cannata, G. Junker and J. Trost, Phys. Lett. A246, 219 (1998); B. Bagchi and R. Roychoudhury, J. Phys. A33, L1 (2000).

[3] A. Ushveridze, Quasi-Exactly Solvable Models in Quantum Mechanics, Inst. of Physics Publishing, Bristol, (1994), and references their in.

[4] C.M. Bender and A. Turbiner, Phys. Lett. A173, 442 (1993).

[5] A. Khare and B.P. Mandal, J. Math. Phys. 39, 3476 (1998).

[6] C. M. Bender and G. V. Dunne, J. Math. Phys. 37 (1996) 6.

[7] A. Khare and B. P. Mandal, quant-ph/0004019.

[8] F. Finkel, A . Gonzaler-lopez and M.A. Rodriguez, J. Math. Phys. 40 (1999) .

[9] A. Krajewska, A. Ushveridze and Z. Walczak, Mod. Phys. Lett. A 12 (1997) 1225. 